\title{
CMB Temperature Polarization Correlation and Primordial Gravitational Waves: WMAP5
}

\author{
N. J. Miller, ${ }^{1}$ B. G. Keating, ${ }^{1}$ and A. G. Polnarev ${ }^{2}$ \\ ${ }^{1}$ Center for Astrophysics and Space Sciences, University of California, San Diego, 9500 Gilman Drive, La Jolla, CA 92093, USA \\ ${ }^{2}$ Astronomy Unit, School of Mathematical Sciences, Queen Mary, University of London, Mile End Road, London E1 4NS, UK
}

Correspondence should be addressed to N. J. Miller, nmiller@physics.ucsd.edu

Received 18 November 2008; Revised 29 December 2008; Accepted 2 February 2009

Recommended by William Reach

we continue our study of the CMB temperature polarization (TE) cross-correlation as a source of information about primordial gravitational waves (PGWs). In a previous paper, we considered two methods for detecting PGWs using the TE cross-correlation. The first method is the zero multipole method, where we find the multipole, $\ell_{0}$, where the TE cross-correlation power spectrum, $C_{\ell}^{T E}$, first changes sign. The second method Wiener filters the CMB TE data to remove the density perturbation contribution to the TE power spectrum. We then use statistical tests to determine if there is a detection of negative residual TE correlation and hence a detection of primordial gravitational waves, the only source of negative TE correlation at these superhorizon scales. In this paper, we will apply these tests to the WMAP 5-year data. We find that the TE power spectrum consistent with $r<2.0$ at $95 \%$ confidence with no additional assumptions about the PGWs. If we assume that the PGWs are generated by inflation, then we get $r<1.0$ at $95 \%$ confidence.

Copyright ( 2009 N. J. Miller et al. This is an open access article distributed under the Creative Commons Attribution License, which permits unrestricted use, distribution, and reproduction in any medium, provided the original work is properly cited.

\section{Introduction}

The cosmic microwave background (CMB) has provided a wealth of information about cosmological phenomena. The $\mathrm{CMB}$ temperature anisotropy has been measured extremely accurately by the WMAP team over the past five years [1]. The parameters describing the standard $\Lambda C D M$ model have been measured to outstanding precision. The current focus of $\mathrm{CMB}$ telescopes is to measure the primordial gravitational wave (PGW) background. Most attention has been focused on detecting the $\mathrm{BB}$ power spectrum of the $\mathrm{CMB}$ which can only be generated by PGWs at large scales. In the previous paper [2], we considered a different approach and looked at how well the TE power spectrum could constrain PGWs. In this paper, we will apply the methods formulated in [2] to the publically available WMAP5 data [3].

PGWs (tensor perturbations) generate negative temperature-polarization (TE) correlation for low multipoles, while primordial density (scalar) perturbations generate positive TE correlation for low multipoles (see $[2,4-6]$ ). This signature can be used to detect or constrain the amount of PGWs. The tests formulated from this signature are useful as an insurance against false detection of PGWs using the
BB power spectrum or as a way to monitor imperfectly subtracted systematic effects and foregrounds.

In this paper, we apply these tests to the WMAP5 data, to see how well PGWs can be detected or constrained using real data. By detection of PGWs, we mean the measurement of the parameter $r$ defined as the ratio of the primordial tensor power spectrum, $P_{t}(k)$, to the primordial scalar power spectrum, $P_{s}(k)$, measured at the wave number $k_{0}=$ $0.002 \mathrm{Mpc}^{-1}$.

The plan of this paper is as follows. In Section 2, we will give a brief description of the two methods based on the TE cross-correlation which can detect or constrain PGWs. In Section 3, we will apply these two methods to the WMAP5 data and discuss the results.

\section{TE Cross-Correlation and Detection of PGWs}

In [2], we looked at two different methods for detecting PGWs based on the TE cross-correlation. The first method, called the zero multipole method, is based on the calculation of the lowest $\ell$, where $C_{\ell}^{\mathrm{TE}}=0$. This value, denoted as $\ell_{0}$, will be smaller for larger $r$. To find $\ell_{0}$, the TE cross-correlation 
is approximated around $\ell_{0}$ by a linear dependence on $\ell$. We can use observed data to fit this linear dependence. Finally, $\ell_{0}$ is determined as the crossing of this fitted line with the $\ell$ axis. The errors on $\ell_{0}$ are calculated by using a Monte Carlo simulation. We generate many mock datasets, using the best fits $C_{\ell}$ 's and the errors provided by the WMAP5 data for the TE cross-correlation. We then calculate $\ell_{0}$ for each of these datasets. The statistical deviation in these $\ell_{0}$-values is considered as the uncertainty in the determination of $\ell_{0}$.

The second method we look at is what we called in [2] and will call hereafter, for brevity, the Wiener filtering method. In actuality, the operator employed is the square root of the Wiener filter, as described in [7]. It has been used before, where it was called "power filtration", in [8]. We initially filter out the contributions to the TE power spectrum due to density perturbations. We then use several nonparametrical statistical tests to determine if there is a residual nonzero TE power spectrum due to PGWs. The statistical tests used are (a) the sign test, (b) a signal-to-noise test, and (c) the Wilcoxon rank-sum test.

(a) The sign test is applied to the tensor contribution to the TE cross-correlation power spectrum, denoted as $C_{\ell, t}^{\mathrm{TE}}(t$ for tensor). If $r=0$, then $C_{\ell, t}^{\mathrm{TE}}=0$ for all $\ell$. If the measured data are equally distributed around $C_{\ell, t}^{\mathrm{TE}}=0$ as one would expect, then there should be roughly an equal number of positive and negative measured values. If $r>0$, then there will be more negative measured values than positive.

(b) The signal-to-noise test deals with the sum of the signal-to-noise ratios for many $\ell$. If, by definition, $S / N=$ $\sum_{i} C_{\ell_{i}} / \Delta C_{\ell_{i}}$, then a negative value of $S / N$ is a signature of PGWs in the data.

(c) The Wilcoxon rank sum test requires a second mock dataset. For this test, we combine the $C_{\ell, t}^{\mathrm{TE}}$ from both the WMAP5 data and the mock data into one large dataset, which contains $m$ multipoles. Then, we rank all multipoles in the large data set from (1) to $m$. The variables $R_{1}$ and $R_{2}$ are defined as the sums of the ranks for the first original dataset and the second original dataset, correspondingly. Then, we introduce the variables $U$ and $U_{i}$ as

$$
\begin{aligned}
U & =\min \left(U_{1}, U_{2}\right), \\
U_{i} & =R_{i}-n_{i}\left(n_{i}+1\right) / 2,
\end{aligned}
$$

where $n_{i}$ is the number of multipoles in that dataset $(i=$ $1,2)$. The distribution of $U$ is known if $r=0$. Its value is used to determine the probability that the two datasets do not have the same $r$. For a more detailed discussion of these two methods for detection of PGWs using the TE cross correlation and the statistical tests see [2, Sections 3 and 4].

\section{Discussion and Results}

We apply the test described in Section 2 to the publically available WMAP5 data. We use both the binned and unbinned versions of the data. The unbinned version of the data provides values and errors for every multipole. The binned version of the data provides fewer measurements, but smaller error bars because binning means the averaging over $\ell$ from $\ell$ to $\ell+\Delta \ell$. The current best constraint on $r$ is

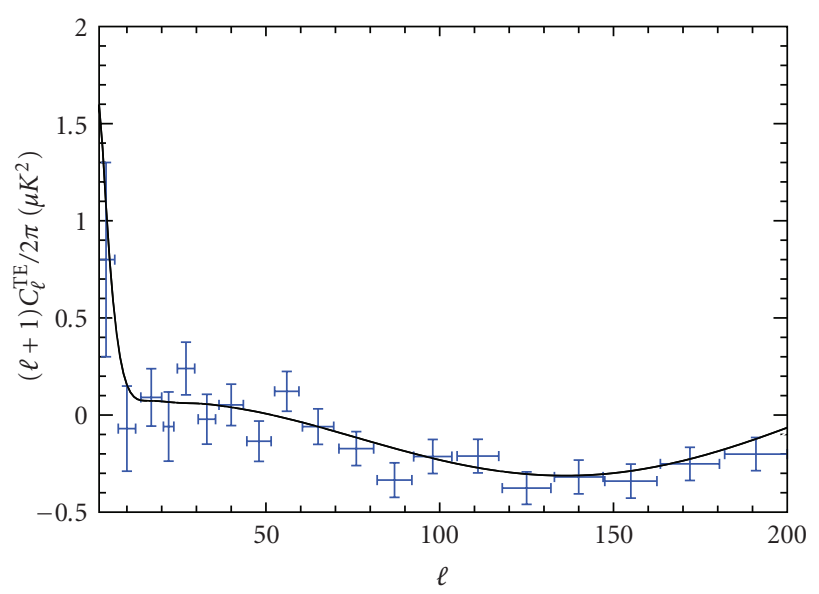

Figure 1: The solid black line is the TE cross-correlation power spectrum predicted by the $\Lambda C D M$ model with no PGWs, which is the best fit for all WMAP5 data. This plot also shows WMAP5 results for TE cross-correlation with error bars (blue crosses).

$r<0.2$ at $95 \%$ confidence level. This constraint is provided by WMAP5 in combination with distance measurements of Type Ia supernovae and imprints of baryon acoustic oscillations on the spatial distribution of galaxies ([9]). By itself, WMAP5 provides a constraint of $r<0.43$ at $95 \%$ confidence level [10]. The $\Lambda C D M$ model of WMAP5 gives $\ell_{0}=52$. A plot of the WMAP5 best fits within the framework of the $\Lambda C D M$ model, and their results of $C_{\ell}^{\mathrm{TE}}$ measurements are shown in Figure 1.

We use several different fitting routines to determine the value of $\ell_{0}$ from the WMAP5 data.

(a) The first routine is a linear fit. This routine minimizes the $\chi^{2}$ error criterion. In other words, this is the least squares technique. We minimize the value $S_{1}=\sum_{i}\left(f\left(\ell_{i}\right)-C_{\ell_{i}}\right)^{2}$, where $f$ is our linear fitting function, and $\ell_{i}$ and $C_{\ell_{i}}$ are the data.

(b) The second routine, which is also a linear fit, is a least absolute deviations fitting routine. Instead of minimizing $S_{1}$, we minimize $S_{2}=\sum_{i}\left|f\left(\ell_{i}\right)-C_{\ell_{i}}\right|$. An advantage to this routine is that it should be more robust to outlying data compared to the least squares technique. A disadvantage is that it is unstable. By instability, we mean that small variations in $\ell$ can cause considerable variations in the slope of the fitted line. In contrast, the least squares technique is stable. The second disadvantage is that the minimum of $S_{2}$ may correspond to more than one fitting line.

(c) Our third fitting routine, in contrast to both previous ones, is a polynomial fit rather than linear. We use the same least squares fitting routine as our first routine, except we fit to a quadratic polynomial instead of a linear one.

For all our routines, we fit over the range $\ell=35$ to $\ell=$ 70. The TE correlations can be approximated as a line over this range for our first two routines, and $\ell_{0}$ will be within this range unless $r$ is extremely large. 


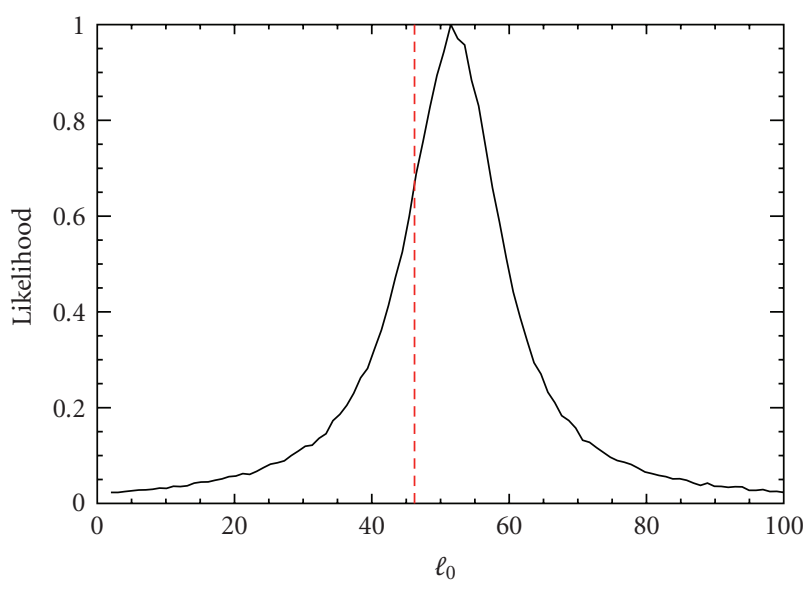

(a)

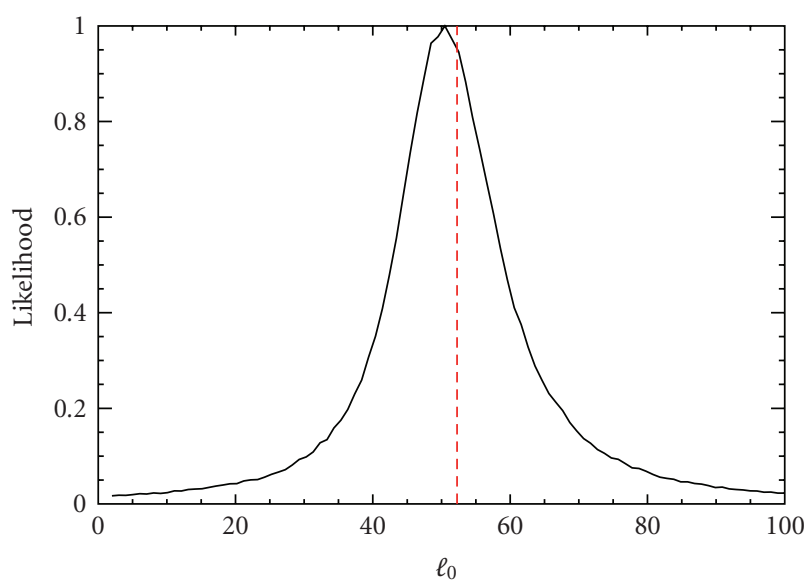

(c)

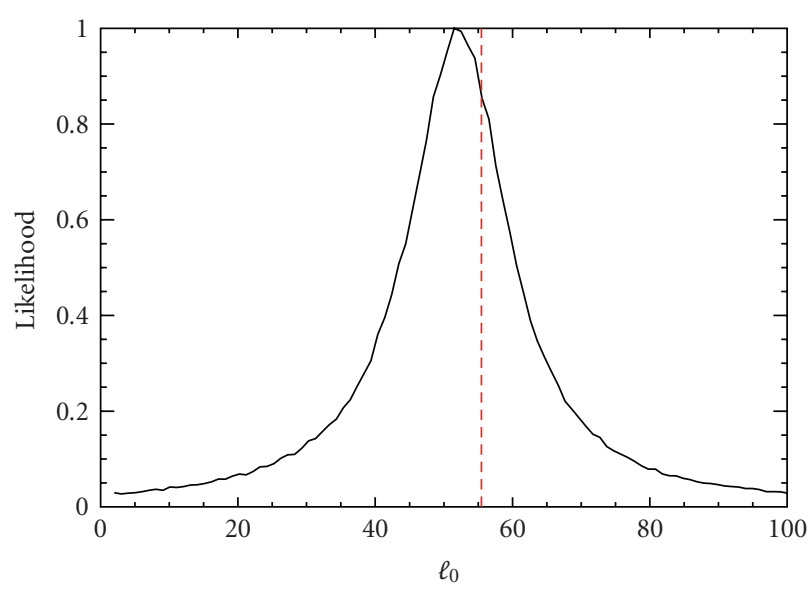

(b)

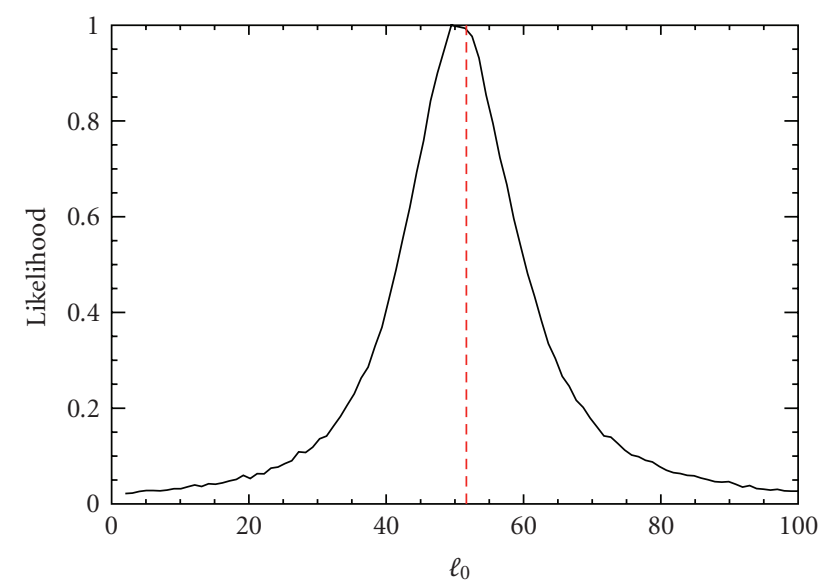

(d)

FIgURE 2: These plots show the results of the calculation of $\ell_{0}$ for the two linear fitting routines. The top row is for the unbinned data, and the bottom row is for the binned data. The left column is the routine which minimizes the $\chi^{2}$ error criterion. The right column is the least absolute deviation fitting routine.

The results for the calculation of $\ell_{0}$ for the two linear fitting routines are shown in Figure 2, and the result for the polynomial fitting routine is shown in Figure 3. A table of the calculated $\ell_{0}$ and their standard deviation is shown in Table 1. There is no detection of PGWs using this method. The measured values are all consistent with $\ell_{0}=52$. To go from a limit on $\ell_{0}$ to a limit on $r$, we first calculate the $\ell_{0}$ as a function of $r$ and $n_{t}$. We then marginalize over $n_{t}$ by integrating the likelihood function to remove the dependance on $n_{t}$. Doing this, we get a limit of $r<2.0$ at $95 \%$ confidence. If we use the assumption that the PGWs are generated by inflation and apply the inflationary consistency relation, $n_{t}=-r / 8$ [11], we get $r<1.0$ at $95 \%$ confidence. Neither of these is better than $r<0.43$ at $95 \%$ confidence.

Applying the filtering method, we assume that the power spectrum of scalar perturbations is given by the $\Lambda C D M$ model, which is the best fit to the WMAP5 data. Then, we apply the statistical tests (a), (b), and (c) to the difference between the power spectrum of this model and power spectrum formed from the raw data.

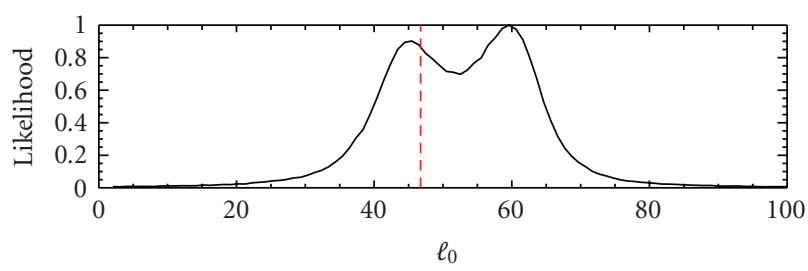

(a)

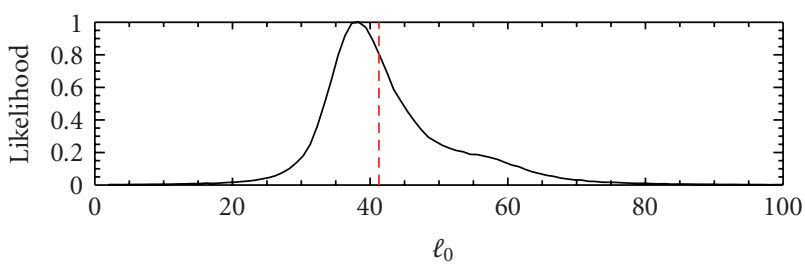

(b)

FIGURE 3: These plots show the results of the calculation of $\ell_{0}$ for the polynomial fitting routine. The top plot is for the unbinned data, and the bottom plot is for the binned data. 


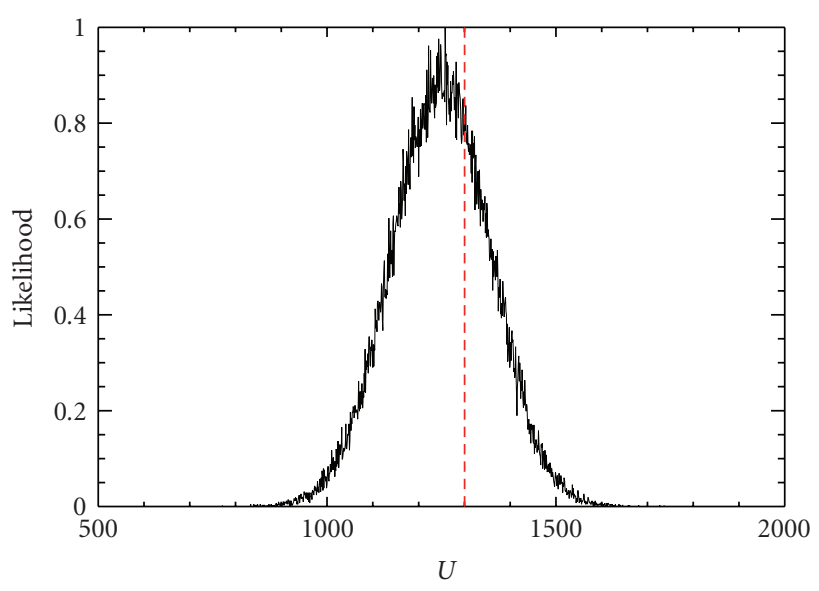

(a)

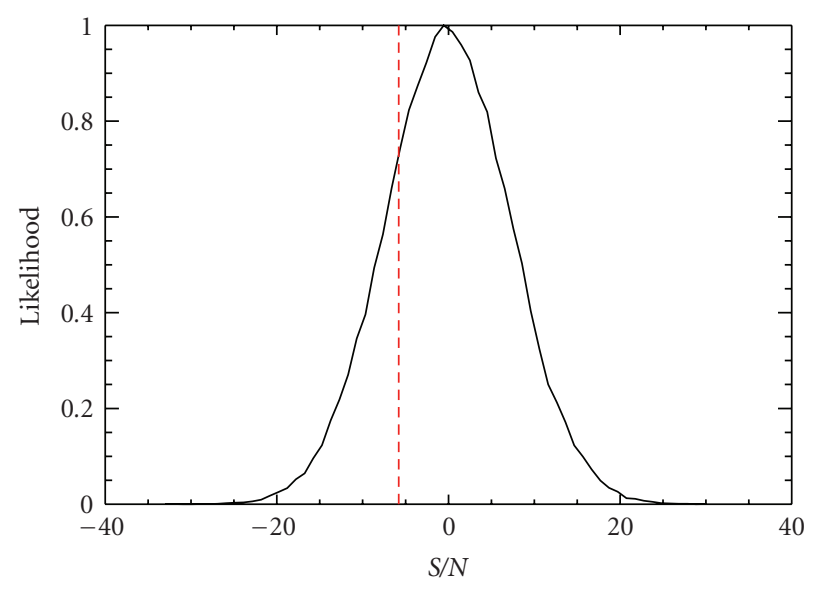

(c)

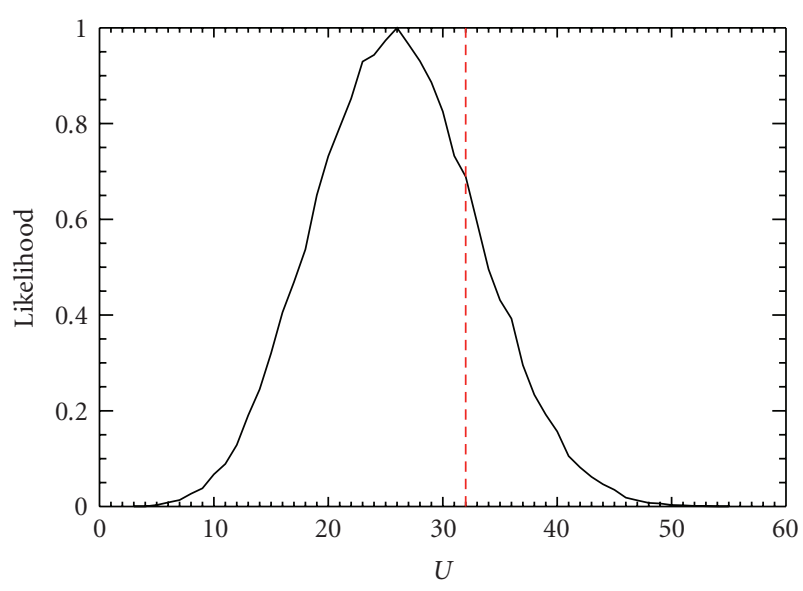

(b)

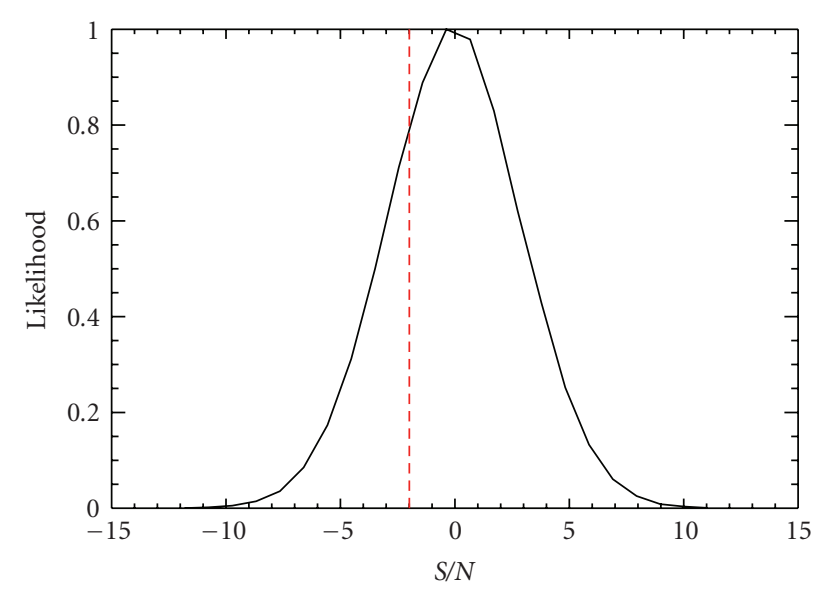

(d)

FIgURE 4: The upper left plot shows the results for the Wilcoxon rank sum test using the unbinned data. The dashed red line shows the mean value of $U$ if $r=0$. The solid black line is the distribution of $U$ with the WMAP5 dataset and a mock dataset with WMAP5 error bars and $r=0$. The upper right plot is the same as the upper left plot except it uses the binned data. The lower left plot shows the results for the $S / N$ test using the unbinned data. The dashed red line is the value of $S / N$ for WMAP5. The solid black line is the distribution of the $S / N$ value for a mock dataset with $r=0$ and WMAP5 error bars. The lower right plot is the same as the lower left plot except it uses the binned data.

TABle 1: A table of the zero multipole, $\ell_{0}$, and the uncertainty in $\ell_{0}, \Delta \ell_{0}$, for the different fitting routines and datasets considered in Section 3

\begin{tabular}{lccc}
\hline Fitting & Data & $\ell_{0}$ & $\Delta \ell_{0}$ \\
\hline linear, $\chi^{2}$ & Unbinned & 46.2 & 14.2 \\
linear, abs. dev. & Unbinned & 55.5 & 14.8 \\
quadratic, $\chi^{2}$ & Unbinned & 46.8 & 11.2 \\
linear, $\chi^{2}$ & Binned & 52.26 & 13.6 \\
linear, abs. dev. & Binned & 51.7 & 14.3 \\
quadratic, $\chi^{2}$ & Binned & 41.3 & 10.1 \\
\hline
\end{tabular}

The results of the filtering are shown in Figure 4. For the Wilcoxon rank sum test, the plots show the mean value of $U$ if $r=0$ in the WMAP5 dataset and the mock dataset (red dashed line) and the distribution of $U$ given the WMAP5 dataset and a mock dataset with $r=0$ (solid black line). Results using both the unbinned WMAP5 data along with the binned WMAP5 data are shown in Figure 4. For the
Wilcoxon rank sum test, the distribution of $U$ is centered somewhat below the mean value we would get if $r=0$ in the WMAP5 data. The difference is only $0.34 \sigma$ for the unbinned data and $0.6 \sigma$ for the binned data, so we cannot reject the hypothesis that $r=0$. The $S / N$ values are less than half of a standard deviation away from a value of zero, which they would be if the TE power spectrum due to density perturbations matched perfectly to the measured TE power spectrum. We cannot place a limit on $r$ using these tests because they are only testing hypotheses that $r=0$. Since these are nonparametric statistical tests, they can help to answer the question whether $r=0$ or not. In other words, all these tests do not help constrain $r$.

\section{Conclusion}

The large angular scale part of the CMB TE power spectrum can be used to constrain PGWs. The WMAP5 data was chosen as WMAP5 has made the best measurements of the 
TE power spectrum so far at the scales we are looking at. QUAD has also made high-sensitivity measurements of the TE power spectrum, but on smaller scales [12] We used two different methods for detecting PGWs. Neither technique was able to detect PGWs in the released data as expected. We are able to say that $r<2.0$ at 95\% confidence, which is nowhere near the current $r<0.43$ limit at $95 \%$ confidence provided by WMAP 5 alone. Using this technique, it will be impossible to improve this constraint. As shown in [2], a cosmic variance limited experiment would only be able to set a limit of $r<0.3$ at $99 \%$ confidence or $r<0.2$ at $95 \%$ confidence from TE data. In other words, a cosmic variance limited experiment could set constraints using these technique that are similar to current constraints provided by WMAP using different techniques. This is also similar to the constraints provided by a cosmic variance limited detection of just the TT power spectrum [13]. These techniques, however, are helpful, as mentioned above, as insurance against a false detection of $r$ from such effects as beam systematics $[14,15]$. These effects could be potential pitfalls for upcoming experiments with higher signal-to-noise than WMAP. In the future, we will consider the insurance against false detection separately and in more detail. For this purpose, we supplement TE cross-correlation measurements with large scale structure data, pulsar timing measurements, baryon acoustic oscillations, supernovae measurements, and so on.

\section{Acknowledgments}

B. G. Keating gratefully acknowledges support from NSF PECASE Award AST-0548262. The authors acknowledge P. Naselsky and K. Griest for their important comments and references.

\section{References}

[1] M. R. Nolta, J. Dunkley, R. S. Hill, et al., "Five-year Wilkinson Microwave Anisotropy Probe (WMAP) observations: angular power spectra," Astrophysical Journal Supplement, vol. 180, no. 2, pp. 296-305, 2009.

[2] A. G. Polnarev, N. J. Miller, and B. G. Keating, "CMB temperature polarization correlation and primordial gravitational waves," Monthly Notices of the Royal Astronomical Society, vol. 386, no. 2, pp. 1053-1063, 2008.

[3] http://lambda.gsfc.nasa.gov/product/map/current.

[4] D. Baskaran, L. P. Grishchuk, and A. G. Polnarev, "Imprints of relic gravitational waves in cosmic microwave background radiation," Physical Review D, vol. 74, no. 8, Article ID 083008, 32 pages, 2006.

[5] R. G. Crittenden, D. Coulson, and N. G. Turok, "Temperaturepolarization correlations from tensor fluctuations," Physical Review D, vol. 52, no. 10, pp. R5402-R5406, 1995.

[6] L. P. Grishchuk, "Discovering relic gravitational waves in cosmic microwave background radiation," http://arxiv.org/ abs/0707.3319.

[7] S. V. Vaseghi, Advanced Digital Signal Processing and Noise Reduction, John Wiley \& Sons, New York, NY, USA, 2006.

[8] D. I. Novikov, P. Naselsky, H. E. Jørgensen, P. R. Christensen, I. D. Novikov, and H. U. Nørgaard-Nielsen, "Power filtration of CMB observational data," International Journal of Modern Physics D, vol. 10, no. 2, pp. 245-259, 2001.

[9] E. Komatsu, J. Dunkley, M. R. Nolta, et al., "Five-year Wilkinson Microwave Anisotropy Probe (WMAP) observations: cosmological interpretation," Astrophysical Journal Supplement, vol. 180, no. 2, pp. 330-376, 2009.

[10] J. Dunkley, E. Komatsu, M. R. Nolta, et al., "Five-year Wilkinson Microwave Anisotropy Probe (WMAP) observations: likelihoods and parameters from the WMAP data," Astrophysical Journal Supplement, vol. 180, no. 2, pp. 306-329, 2009.

[11] M. Kamionkowski and A. Kosowsky, "Detectability of inflationary gravitational waves with microwave background polarization," Physical Review D, vol. 57, no. 2, pp. 685-691, 1998.

[12] C. Pryke, P. Ade, J. Bock, et al., "Second and third season QUaD CMB temperature and polarization power spectra," Astrophysical Journal, vol. 692, no. 2, pp. 1247-1270, 2009.

[13] L. Knox and M. S. Turner, "Detectability of tensor perturbations through anisotropy of the cosmic background radiation," Physical Review Letters, vol. 73, no. 25, pp. 3347-3350, 1994.

[14] N. J. Miller, M. Shimon, and B. G. Keating, "CMB beam systematics: impact on lensing parameter estimation," Physical Review D, in press (2009).

[15] M. Shimon, B. Keating, N. Ponthieu, and E. Hivon, "CMB polarization systematics due to beam asymmetry: impact on inflationary science," Physical Review D, vol. 77, no. 8, Article ID 083003, 11 pages, 2008. 

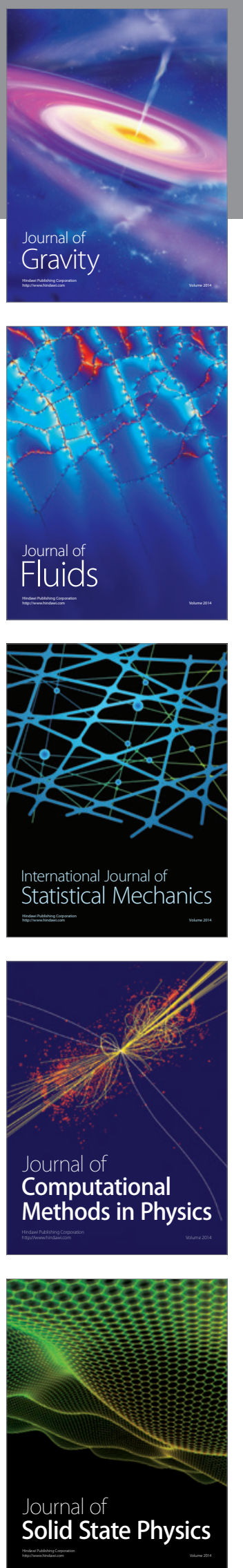

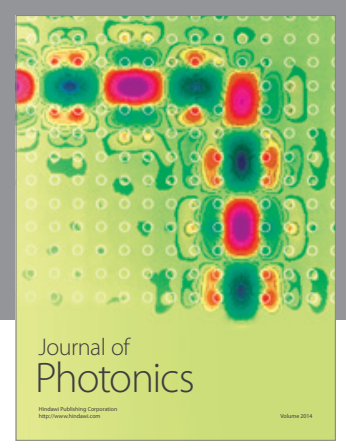

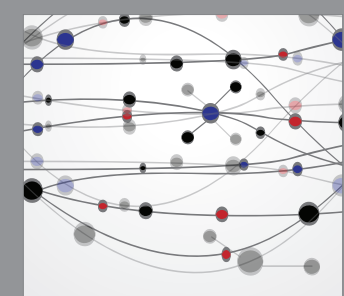

The Scientific World Journal
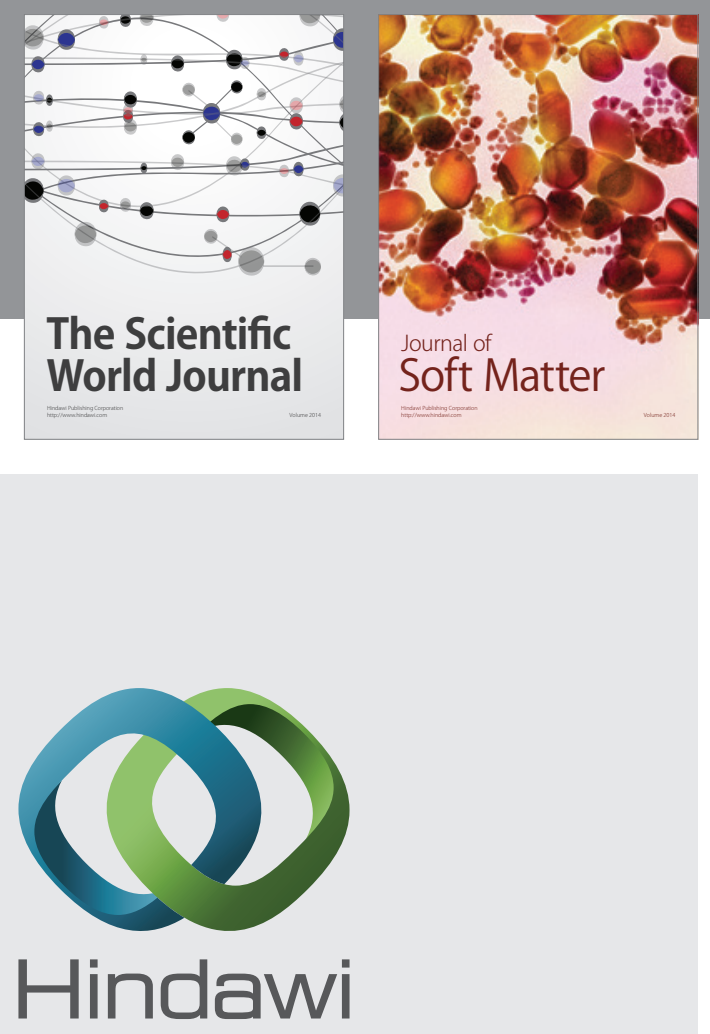

Submit your manuscripts at

http://www.hindawi.com
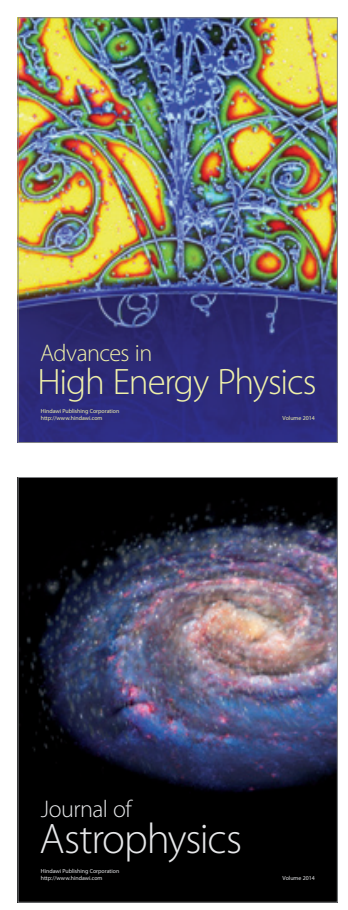
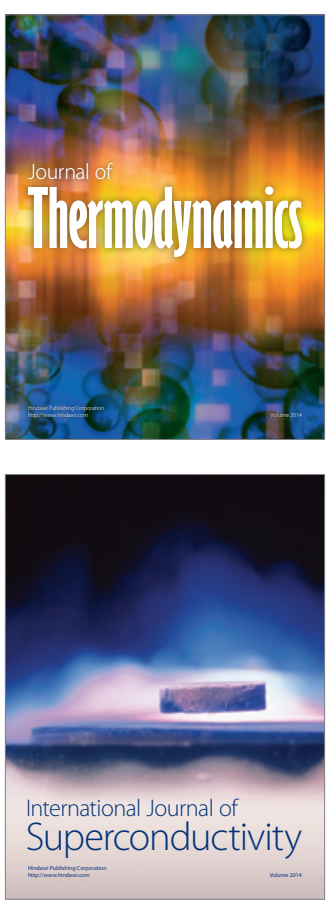
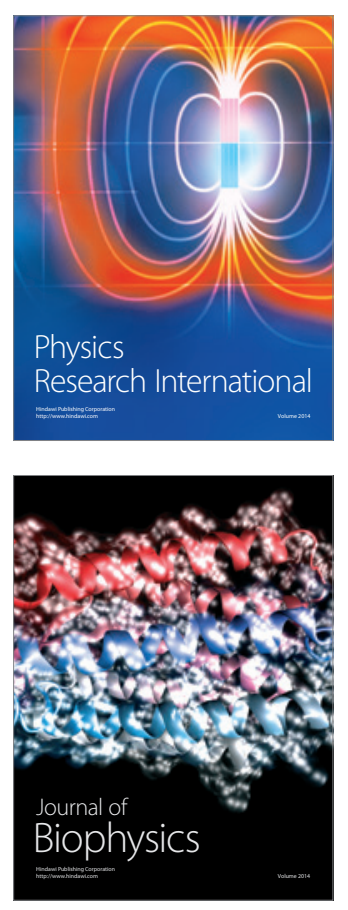
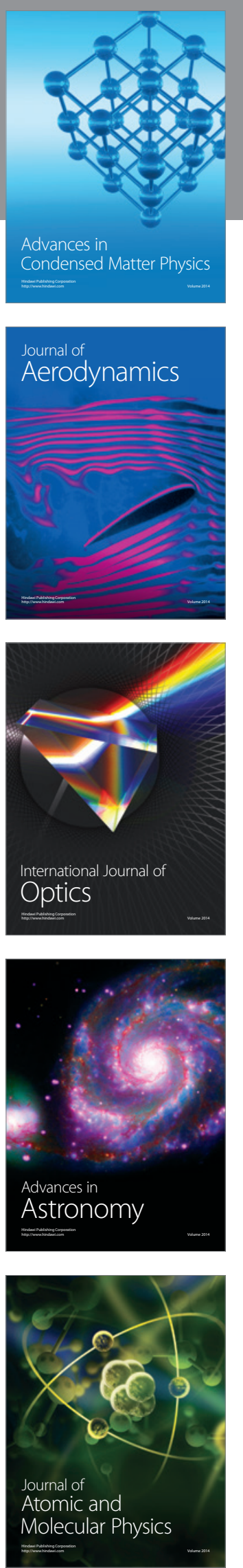